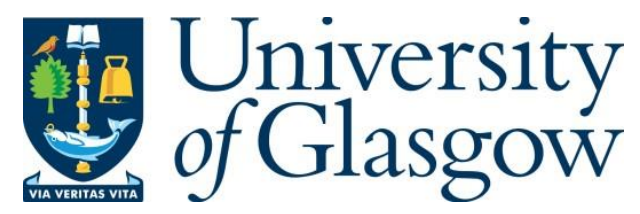

Colonia, S., Steijl, R. and Barakos, G. (2018) Shock interactions in continuum and rarefied conditions employing a novel gas-kinetic scheme. In: Kontis, K. (ed.) Shock Wave Interactions: Selected Articles from the 22nd International Shock Interaction Symposium, University of Glasgow, United Kingdom, 4-8 July 2016. Springer, pp. 107122. ISBN 9783319731797.

There may be differences between this version and the published version. You are advised to consult the publisher's version if you wish to cite from it.

http://eprints.gla.ac.uk/159794/

Deposited on: 29 March 2018

Enlighten - Research publications by members of the University of Glasgow http://eprints.gla.ac.uk 


\title{
Shock Interactions in Continuum and Rarefied Conditions Employing a Novel Gas-Kinetic Scheme
}

\author{
S. Colonia, R. Steijl and G. Barakos
}

\begin{abstract}
Shock interactions can have a significant impact on heating rates and aerodynamic performance of hypersonic vehicles. The study presents different shock interactions in partially-rarefied hypersonic flows predicted employing a recently developed gas-kinetic scheme for diatomic gases with rotational degrees of freedom. The new gas-kinetic schemes will be presented along with shock/wave boundary interactions as well as Edney Type IV shock-shock interactions. Various levels of rarefaction have been considered to highlight the effect of thermal relaxation between the translational and rotational modes. In addition, for the Edney test case the imposed wall temperature on the shock-generating wedge and the cylinder surface has been varied, to evaluate the importance of the boundary layer thickness on the interaction region.
\end{abstract}

\section{Introduction}

The understanding of shock interactions is critical to the design of hypersonic vehicles since they can have a significant impact on both heating rates and aerodynamic performance affecting the requirements for vehicle control and thermal protection. The flow over deflected control surfaces may be dominated by shock wave/boundary layer interactions that can cause extensive separation, a laminar-to-turbulence tran-

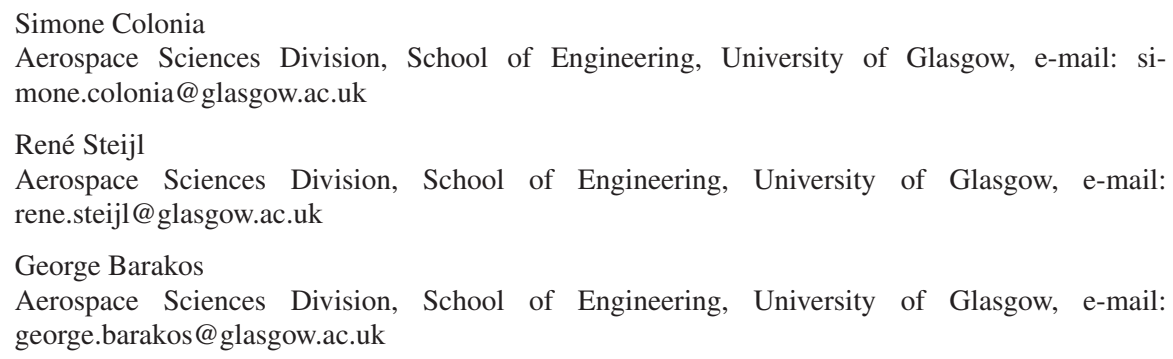


sition and localised intense heating. A recent review of some of the key findings for shock wave/boundary layer interactions for laminar flows was presented in [1], where it was shown that mechanical and thermal loads over compression ramps depend strongly on ramp angle, wall temperature and geometric configuration. Furthermore, the dependency of numerical predictions on grid resolution and the correctness of the employed physical and numerical modelling was addressed.

In the present work, shock interactions in partially-rarefied hypersonic flows are considered. For the efficient simulation of such flows, a number of different approaches can be considered. One such approach involves hybrid techniques which couple Navier-Stokes methods with DSMC solvers or discrete-velocity methods (DVM) for kinetic Boltzmann equations. In these methods, the more expensive approach, DSMC or DVM, is employed only where needed and is coupled with a finite-volume scheme for the NS equations used where the flow is continuum. A hybrid technique couples two different simulation methods by means of information exchange between the parts of the flow domain. Recent works using these methods focused on rarefied high-Mach flow include [2] and [3]. Early results for hybrid rarefied high-Mach simulations using the Multi-Physics Code (MФC) used in the present work were reported in [4].

An alternative approach involves gas-kinetic schemes originally introduced as Navier-Stokes solvers, which more recently have been extended with a capability to resolve thermal non-equilibrium and flow rarefaction effects. In the present work, a recently developed gas-kinetic scheme for diatomic gas flows with rotational relaxation is employed in the analysis of flows with shock interactions. The strength of the gas-kinetic scheme lies in its capability to include the effect of collisions introducing a multi-scale effect which is missing in more commonly-used Navier-Stokes schemes. Moreover, due to their nature, gas-kinetic schemes can predict flow fields with a certain degree of rarefaction enabling the computational analysis of shock interactions from the continuum to the rarefied regime without having to rely on different numerical methods. In gas-kinetic schemes, a finite-volume approach is used which reconstructs local kinetic problems around the cell interfaces in order to calculate the numerical fluxes. In contrast, more commonly used Roe or AUSM fluxes are based on approximations to a local continuum Riemann problem. In the present work, the local kinetic problem around the cell interfaces includes both convective and viscous contributions, while finite-volume methods employing more commonly-used numerical flux methods, e.g. Roe or AUSM, for the convective fluxes typically employ a separate central difference-based discretisation of the viscous fluxes.

Among the gas-kinetic schemes available in the literature, a successful approach is represented by the BGK-NS method introduced by [5], subsequently improved resulting in the Unied Gas Kinetic Scheme (UGKS) by [6] and extended to diatomic gas flows in [7]. The Unified Gas-Kinetic Scheme (UGKS) uses a finitevolume method where the numerical fluxes are based on the solution of the Shakhov model [8] for a monoatomic gas, or the Rykov model [9] for a diatomic gas with rotational non-equilibrium. Where the flow is under-resolved, by accounting for the pressure jump in the definition of the collision time, additional numerical viscosity 

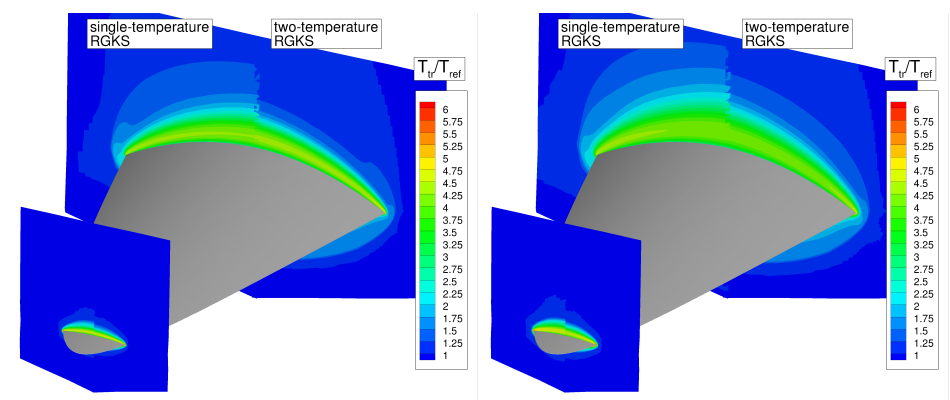

Fig. 1 Mach 8 flow around waverider with rounded leading edge. Translational temperature in two cross-sections in RGKS simulations. RGKS model with rotational relaxation on right-hand side, single-temperature RGKS result on left-hand side. Two Knudsen numbers are shown, $K n=0.0005$ (left) and $K n=0.001$ (right) based on fuselage length.

is added resulting in a shock thickness of the order of the cell size. This allows the UGKS to simulate flows in both rarefied and continuum regimes. A further feature is the reconstruction and storage of the particle distribution functions around the cell faces. which are updated in time according to the considered kinetic model. The means that the UGKS can deal with significant levels of flow rarefaction, but at a memory cost between that of Navier-Stokes gas-kinetic schemes and of discretevelocity methods.

Recently, the authors developed a novel gas-kinetic scheme based on a ChapmanEnskog reconstruction of the kinetic problems around the cell interfaces which includes rotational relaxation consistent with the Rykov model, as published in [10]. The method does not store and update the particle distribution functions in statespace for each cell face, and it is therefore computationally more efficient that the UGKS, particularly in memory overhead. However, the Chapman-Enskog reconstruction limits the scheme to lower levels of rarefaction than the UGKS. Compared to earlier works of $\mathrm{Xu}$ and co-workers the presented scheme is defined directly on the basis of kinetic models which involve a Prandtl number correction.

In the current work an improved version of the gas-kinetic scheme presented by [10] is used which involves a further efficiency improvement by replacing numerical evaluations of moments in state-space with analytically derived expressions. Furthermore, a modified GKS scheme is also considered in which equilibrium between the translational and rotational modes is assumed. Analysing the results of the different schemes will enable the assessment of the effect of thermal relaxation as well as the effect of changing Reynolds number which typically occurs when changing the Knudsen number for a fixed Mach number. The methods were designed for complex three-dimensional flow simulations, including flows around waverider geometries and moderate rarefaction levels, as shown in Figure 1. For increasing Knudsen numbers, the translational temperature from the gas-kinetic scheme based on the Rykov model can be seen to deviate further from the results with the singletemperature gas-kinetic scheme, as could be expected. Considering the good compu- 
tational efficiency of the gas-kinetic scheme derived from the Rykov model (RGKS), a major point of investigation is the determination of what level of flow rarefaction the results are accurate and when a switch to more expensive method such as DSMC or DVM would be needed. In the present work, the assessment of the schemes will first be considered for two-dimensional shock interaction test cases.

The new gas-kinetic schemes will be presented along with the application to hypersonic flows with shock/wave boundary interactions as well as hypersonic flow with Edney Type IV shock-shock interactions. Different levels of flow rarefaction are considered, highlighting the effect of thermal relaxation between the translational and rotational modes in the considered diatomic gases. Furthermore, the sensitivity of the shock-interaction with respect the imposed wall temperature on the shock-generating wedge and the cylinder surface was considered, highlighting the importance of the boundary layer displacement thickness increase on the wedge.

\section{Multi-Physics Code}

The methods used in the present work are built in the Multi-Physics Code (MФC) under development at the University of Glasgow, described in [11] and [10]. МФC is a computational framework designed for simulations of complex flows, where different mathematical flow models are employed for different regions of the flow domain depending on the flow physics. For compressible-flow applications, a cellcentred block-structured finite-volume method for the Navier-Stokes equations using the $\mathrm{AUSM}^{+}$/up method for the convective fluxes forms the baseline model. For high-speed flows with thermodynamic non-equilibrium, the solver uses RGKS (gas-kinetic scheme based on the Rykov model), introduced in [10]. For more challenging flows at higher levels of flow rarefaction, discrete-velocity methods for the Shakhov and ES models are available for monoatomic gas flows, while the DVM uses the Rykov model or a poly-atomic ES model for diatomic gas flows. Considering the cost and memory requirement for the DVM, practical applications to highspeed flows involve hybrid simulations coupling the DVM with the NS or RGKS solver. With improved gas kinetic schemes as presented in [10], the extent to which the DVM needs to be used can be reduced in hybrid simulations, and therefore achieve significant efficiency gains. In the present work, the emphasis is on simulations which use the RGKS in the entire computational domain.

\section{Non-dimensional Rykov Model}

A diatomic gas is considered at temperatures at which vibrational degrees of freedom are not significantly excited. Furthermore, we exclude extremely low temperatures, such that we can assume that rotational degrees of freedom can be considered fully excited. In this case the particle distribution function $f(\mathbf{x}, \mathbf{c}, t, \zeta)$, which de- 
scribes the state of the gas, will be a function not only of the spatial coordinate $\mathbf{x}$, the particle velocity $\mathbf{c}$ and the time $t$, but also of the rotational degrees of freedom $\zeta$. The Rykov model represents an extension of the Shakhov model where also rotational non-equilibrium is considered and has been proved to be a reliable kinetic approximation, up to the heat fluxes moments of the Boltzmann equation. Since the rotational degrees of freedom is considered fully excited, $\zeta$ is reduced by the model and a second distribution function is obtained. The non-dimensional distribution functions $F_{0}$ and $F_{1}$ are obtained from the dimensional distribution functions $\hat{F}_{0}$ and $\hat{F}_{1}$ as follows,

$$
F_{0}=\hat{F}_{0} /\left(\rho_{\infty}\left(2 R T_{\infty}\right)^{-3 / 2}\right) ; F_{1}=\hat{F}_{1} /\left(m R T_{\infty} \rho_{\infty}\left(2 R T_{\infty}\right)^{-3 / 2}\right)
$$

For the Rykov model written in terms of $F=m f$ we obtain,

$$
\begin{aligned}
& \frac{\partial F_{0}}{\partial t}+\mathbf{c} \frac{\partial F_{0}}{\partial \mathbf{x}}=\frac{F_{0}^{e q}-F_{0}}{\tau} ; \frac{\partial F_{1}}{\partial t}+\mathbf{c} \frac{\partial F_{1}}{\partial \mathbf{x}}=\frac{F_{1}^{e q}-F_{1}}{\tau} \\
& F_{0}^{e q}=\frac{1}{Z_{r}} F_{0}^{r}+\left(1-\frac{1}{Z_{r}}\right) F_{0}^{t} ; F_{1}^{e q}=\frac{1}{Z_{r}} F_{1}^{r}+\left(1-\frac{1}{Z_{r}}\right) F_{1}^{t} \\
& F_{0}^{r}=F_{M}(T)\left[1+\frac{8}{15} \omega_{0} \frac{q_{x}^{t}}{p} \frac{c^{\prime}{ }_{x}}{T}\left(\frac{\mathbf{c}^{\prime 2}}{T}-\frac{5}{2}\right)\right] \\
& F_{0}^{t}=F_{M}\left(T_{t}\right)\left[1+\frac{8}{15} \frac{q_{x}^{t}}{p_{t}} \frac{c_{t}^{\prime}{ }_{x}}{T_{t}}\left(\frac{\mathbf{c}^{\prime 2}}{T_{t}}-\frac{5}{2}\right)\right] \\
& F_{1}^{r}=T F_{M}(T)\left[1+\frac{8}{15} \omega_{0} \frac{q_{x}^{t}}{p} \frac{c^{\prime}{ }_{x}}{T}\left(\frac{\mathbf{c}^{\prime 2}}{T}-\frac{5}{2}\right)+4 \omega_{1}(1-\delta) \frac{q_{i}^{r} c^{\prime}{ }_{i}}{p T}\right] \\
& F_{1}^{t}=T_{r} F_{M}\left(T_{t}\right)\left[1+\frac{8}{15} \frac{q_{x}^{t}}{p_{t}} \frac{c^{\prime}{ }_{x}}{T_{t}}\left(\frac{\mathbf{c}^{\prime 2}}{T_{t}}-\frac{5}{2}\right)+4(1-\delta) \frac{q_{i}^{r} c^{\prime}{ }_{i}}{p_{t} T_{r}}\right]
\end{aligned}
$$

where the total collision time $\tau$ is expressed as $\mu_{t} / p_{t}$ with the viscosity determined from the translational temperature and $F_{M}$ is the Maxwellian. In the present work the values $\omega_{0}=0.5$ and $\omega_{1}=0.286$ and $\delta=0.75$ are employed. $Z_{r}$ is the collision number, representing the number of collisions between molecules required to force the rotational and translational motions towards equilibrium. In the recent literature, formulas for $Z_{r}$ derived from data fitting, either from numerical or experimental results, have been employed. For the Rykov model, the collision number suggested by Rykov and co-workers is,

$$
\begin{aligned}
& Z_{r}^{R y k .}=\frac{3}{4} \pi \frac{\psi(\tilde{T})}{\tilde{T}^{1 / 6}} \frac{9 \tilde{T}}{\tilde{T}+8} \frac{T_{r}}{T_{t}}\left[0.461+0.5581\left(\frac{T_{r}}{T_{t}}\right)+0.0358\left(\frac{T_{r}}{T_{t}}\right)^{2}\right] \\
& \psi(\tilde{T})=0.767+0.233 \tilde{T}^{-1 / 6} \exp (-1.17[\tilde{T}-1]) ; \tilde{T}=T_{t} / T^{*}
\end{aligned}
$$

$T *=91.5 \mathrm{~K}$ is the characteristic temperature of the intermolecular potential for Nitrogen. An alternative expression for $Z_{r}\left(T_{t}, T_{r}\right)$ derived from molecular dynamics 
simulations can be found in [12] as

$$
Z_{r}^{\text {Val. }}=\left[a_{1}\left(\frac{T_{t}}{1 K}\right)^{1 / 4}+a_{2}\left(\frac{T_{t}}{1 K}\right)^{-1 / 4}-a_{3}\left(\frac{T_{t}}{1 K}-1000\right)\right]\left[1-b\left(1-\frac{T_{r}}{T_{t}}\right)\right]
$$

with $a_{1}=1.33868, a_{2}=-6.19992, a_{3}=-0.00107942$ and $0<b \leq 1$. For the viscosity law, Rykov and co-workers suggest

$$
\mu\left(T_{t}\right)=\mu\left(T^{*}\right) \frac{\tilde{T}^{2 / 3}}{\psi(\tilde{T})}
$$

Alternatively, a simpler power law can be employed. In the present work, this power law was used exclusively, with an exponential factor of 0.72 .

\section{Gas Kinetic Scheme based on Rykov model - RGKS}

The present scheme is analytically defined on the basis of the Chapman-Enskog (CE) expansion of the non-dimensional Rykov model for diatomic gases. The derivatives of the equilibrium function and the time derivatives of the primitive variables are defined analytically employing the compatibility condition of the kinetic model for the latter. In previous works, e.g. [13] similar gas-kinetic schemes are defined using the CE solution of the BGK model with and without rotational nonequilibrium and a scaling of the energy numerical flux defined in [5] to correct the Prandtl number. Moreover, in those schemes the required derivative are expressed in terms of Taylor series where the coefficient are calculated by means of properties of the employed BGK model. The proposed GKS, due to the use of the CE expansion, is limited to near-continuum regions but is simpler than the UGKS methods presented in [6] and [7]. For the present Rykov-based GKS solver, also this correction is defined fully analytically. Results for hypersonic partially-rarefied flows with shocks using this correction were previously presented in [10], showing an improved agreement with experimental data for the obtained shock thickness and profiles. From the non-dimensional reduced Rykov model (2), a system of equations for the vector of non-dimensional macroscopic variables $\mathbf{W}_{\mathbf{R}}=\left(\rho, \rho \mathbf{c}, \frac{5}{2} \rho T+\rho c^{2}, \rho T_{r}\right)^{T}$ is obtained by taking moments $\Psi_{0}=\left(1, \mathbf{c}, c^{2}, 0\right)^{T}$ and $\Psi_{1}=(0, \mathbf{0}, 1,1)^{T}$ and integrating over a time step $\Delta t$. The expressions for the numerical fluxes resulting from this step were previously described in detail in [10]. The moments involve integrations over the velocity space and in most general formulation published previously, numerical evaluation of these integrations was used. In the present work, analytical expressions for the velocity-space integrations were used, which further enhances the efficiency of the proposed gas-kinetic scheme. In the integration, upwinding in the velocity space is used. The full formulation is too long to be presented here. For illustration, the mass flux is presented here only. For the inviscid mass flux, where $l, r$ refer to the left-hand and right-hand side of the cell interface, we find, 


$$
\begin{aligned}
& F^{\text {mass, inviscid }}= \\
& {\left[\frac{\rho_{l}}{2}\left(1+\operatorname{erf}\left(\frac{u_{n, l}}{\sqrt{T_{t, l}}}\right)\right) u_{n . l}+\frac{\rho_{l}}{2} \sqrt{\frac{T_{t, l}}{\pi}} \exp \left(-\frac{u_{n, l}^{2}}{T_{t, l}}\right)\right]\left(1-\frac{1}{Z_{r, l}}\right)+} \\
& {\left[\frac{\rho_{l}}{2}\left(1+\operatorname{erf}\left(\frac{u_{n, l}}{\sqrt{T_{e q, l}}}\right)\right) u_{n . l}+\frac{\rho_{l}}{2} \sqrt{\frac{T_{e q, l}}{\pi}} \exp \left(-\frac{u_{n, l}^{2}}{T_{e q, l}}\right)\right] \frac{1}{Z_{r, l}}+} \\
& {\left[\frac{\rho_{r}}{2}\left(1+\operatorname{erf}\left(\frac{u_{n, r}}{\sqrt{T_{t, r}}}\right)\right) u_{n . r}-\frac{\rho_{r}}{2} \sqrt{\frac{T_{t, r}}{\pi}} \exp \left(-\frac{u_{n, r}^{2}}{T_{t, r}}\right)\right]\left(1-\frac{1}{Z_{r, r}}\right)+} \\
& {\left[\frac{\rho_{r}}{2}\left(1+\operatorname{erf}\left(\frac{u_{n, r}}{\sqrt{T_{e q, r}}}\right)\right) u_{n . r}-\frac{\rho_{r}}{2} \sqrt{\frac{T_{e q, r}}{\pi}} \exp \left(-\frac{u_{n, r}^{2}}{T_{e q, r}}\right)\right] \frac{1}{Z_{r, r}}}
\end{aligned}
$$

The cell-face normal velocity component is $u_{n}$, while $T_{e q}$ is the equilibrium temperature. The Prandtl number correction for the mass flux becomes,

$$
\begin{aligned}
F^{\text {mass }, \text { Pr,corr. }}= & \frac{8}{15}\left[\frac{\rho_{l}}{2} \sqrt{\frac{T_{t, l}}{\pi}} \exp \left(-\frac{u_{n, l}^{2}}{T_{t, l}}\right)\left\{-\frac{q_{n, l} u_{n, l}}{2 \rho_{l} T_{t, l}^{2}}\right\}\left(1-\frac{1}{Z_{r, l}}\right)\right. \\
& +\frac{\rho_{l}}{2} \sqrt{\frac{T_{e q, l}}{\pi}} \exp \left(-\frac{u_{n, l}^{2}}{T_{e q, l}}\right)\left\{-\frac{q_{n, l} u_{n, l}}{2 \rho_{l} T_{e q, l}^{2}}\right\} \frac{\omega_{0}}{Z_{r, l}} \\
& -\frac{\rho_{r}}{2} \sqrt{\frac{T_{t, r}}{\pi}} \exp \left(-\frac{u_{n, r}^{2}}{T_{t, r}}\right)\left\{-\frac{q_{n, r} u_{n, r}}{2 \rho_{r} T_{t, r}^{2}}\right\}\left(1-\frac{1}{Z_{r, r}}\right) \\
& \left.-\frac{\rho_{r}}{2} \sqrt{\frac{T_{e q, r}}{\pi}} \exp \left(-\frac{u_{n, r}^{2}}{T_{e q, r}}\right)\left\{-\frac{q_{n, r} u_{n, r}}{2 \rho_{r} T_{e q, r}^{2}}\right\} \frac{\omega_{0}}{Z_{r, r}}\right]
\end{aligned}
$$

where $q_{n}$ is the heat flux associated with the translational motion normal to the cell interface. For the viscous fluxes, derived from the CE expansion around the local solution on the left-hand and right-hand side of the cell interface, we find,

$$
\begin{aligned}
F^{\text {mass }, \text { viscous }}= & \frac{\rho_{l}}{2} \sqrt{\frac{T_{t, l}}{\pi}} \exp \left(-\frac{u_{n, l}^{2}}{T_{t, l}}\right) \tau_{l}\left[-\frac{u_{n, l}}{T_{t, l}} \frac{\partial T_{t, l}}{\partial n}+\right. \\
& \frac{T_{e q, l}-T_{t, l}}{2 \tau_{l} Z_{r, l} T_{t, l}}+\left(J_{11}^{2}-\frac{1}{3}\right) \frac{\partial u_{l}}{\partial x}+\left(J_{21}^{2}-\frac{1}{3}\right) \frac{\partial v_{l}}{\partial y} \\
& +\left(J_{31}^{2}-\frac{1}{3}\right) \frac{\partial w_{l}}{\partial z}+J_{11} J_{21}\left(\frac{\partial u_{l}}{\partial y}+\frac{\partial v_{l}}{\partial x}\right)+ \\
& \left.J_{11} J_{31}\left(\frac{\partial u_{l}}{\partial z}+\frac{\partial w_{l}}{\partial x}\right)+J_{21} J_{31}\left(\frac{\partial v_{l}}{\partial z}+\frac{\partial w_{l}}{\partial y}\right)\right] \\
& -\frac{\rho_{r}}{2} \sqrt{\frac{T_{t, r}}{\pi}} \exp \left(-\frac{u_{n, r}^{2}}{T_{t, r}}\right) \tau_{r}\left[-\frac{u_{n, r}}{T_{t, r}} \frac{\partial T_{t, r}}{\partial n}+\right.
\end{aligned}
$$




$$
\begin{aligned}
& \frac{T_{e q, r}-T_{t, r}}{2 \tau_{r} Z_{r, r} T_{t, r}}+\left(J_{11}^{2}-\frac{1}{3}\right) \frac{\partial u_{r}}{\partial x}+\left(J_{21}^{2}-\frac{1}{3}\right) \frac{\partial v_{r}}{\partial y} \\
& +\left(J_{31}^{2}-\frac{1}{3}\right) \frac{\partial w_{r}}{\partial z}+J_{11} J_{21}\left(\frac{\partial u_{r}}{\partial y}+\frac{\partial v_{r}}{\partial x}\right)+ \\
& \left.J_{11} J_{31}\left(\frac{\partial u_{r}}{\partial z}+\frac{\partial w_{r}}{\partial x}\right)+J_{21} J_{31}\left(\frac{\partial v_{r}}{\partial z}+\frac{\partial w_{r}}{\partial y}\right)\right]
\end{aligned}
$$

where $J_{11}, \ldots, J_{31}$ represent mesh transformation metrics in the general curvilinear mesh formulation used here. The source term is due to the rotational relaxation. The only non-zero contribution is for the last equation in the system, governing the rotational energy. For the rotational energy equation, the relaxation term has the following form for cell $i$ and integration from time level $n$ to $n+1$,

$$
\frac{\rho\left(\left.T\right|_{i} ^{n+1}-\left.T_{r}\right|_{i} ^{n+1}\right)}{Z_{r} \tau_{i}^{n+1}}+\frac{\rho\left(\left.T\right|_{i} ^{n}-\left.T_{r}\right|_{i} ^{n}\right)}{Z_{r} \tau_{i}^{n}}
$$

where the trapezoidal rule was employed in the time integration. It is important to note that here the difference between the local equilibrium temperature $\left.T\right|_{i}$ and the rotational temperature $\left.T_{r}\right|_{i}$ in cell $i$ is used, while many studies consider a relaxation term of the type introduced by Jeans-Teller involving translational and rotational temperatures. For a well-resolved flow, the exponential terms in the flux equations are small and represent numerical dissipation terms. Using the Rykov model as the basis for the flux formulation, it shows that the Prandtl number correction in Equation (6) combine with the heat-flux related terms in the viscous flux to consistently represent a Prandtl number correction for the part of the heat flux associated with the translational energy.

Based on the RGKS with rotational relaxation, a single-temperature version was also implemented in which it is assumed that the translational and rotational temperatures are in equilibrium, which significantly reduces the complexity of the scheme as can be seen from Equations (5), (6) and(7). For the Rykov kinetic model, the effective Prandtl number for continuum conditions can be derived from a ChapmanEnskog expansion. For the Rykov Gas Kinetic Scheme the Prandtl can be similarly obtained in the limit of the translational and rotational temperatures approaching a common equilibrium temperature. The Prandtl number is defined as,

$$
\frac{1}{\operatorname{Pr}}=\frac{5}{7}\left[\frac{3}{2+\frac{1-\omega_{0}}{Z_{r}}}+\frac{2 / 5}{\delta+\frac{(1-\delta)\left(1-\omega_{1}\right)}{Z_{r}}}\right]
$$

which shows that for the values used in the present work, i.e. $\omega_{0}=0.5, \omega_{1}=0.286$ and $\delta=0.75$, the Prandtl number attains a value of 0.71 for $Z_{r}=5$. In the singletemperature version of the gas-kinetic scheme, $Z_{r}=5$ is used for the flow state at left-hand and right-hand side of the cell interface.

In the present work, solid wall boundary conditions are modelled using diffuse walls with full accommodation. 


\section{Shock wave/boundary layer interaction}

The first test case considered is the laminar flow over a compression ramp with a sharp leading edge. The main point of interest here is the effect of the flow rarefaction on the shock wave/boundary layer interaction. The test case considered was conducted at Calspan-UB Research Center (CUBRC), with a free-stream Mach number of 14.1 and a Reynolds number of 236,200 per meter and a ramp angle of $15^{\circ}$. The free-stream temperature was $88.88 \mathrm{~K}$ and a wall temperature of $297.22 K$. The viscous interaction parameter at the plate/ramp junction is large and for the ramp angle considered, the flow in the experiment stays attached. For larger ramp angles, a separation bubble is formed which is confined by separation and re-attachment shocks, as discussed in detail by [1].

The Knudsen number for this test case is $\mathrm{Kn}=8.85 \cdot 10^{-5}$ for a meter reference length as tested at CUBRC. In the present work, a more rarefied condition is also considered, obtained by scaling down the flow length scale by a factor of two while leaving the inflow conditions unchanged. This doubles Knudsen number and halves the Reynolds number. For this test case, a block-structured mesh with 68 blocks and a total of $10^{6}$ cells in $2 \mathrm{D}$ was employed.

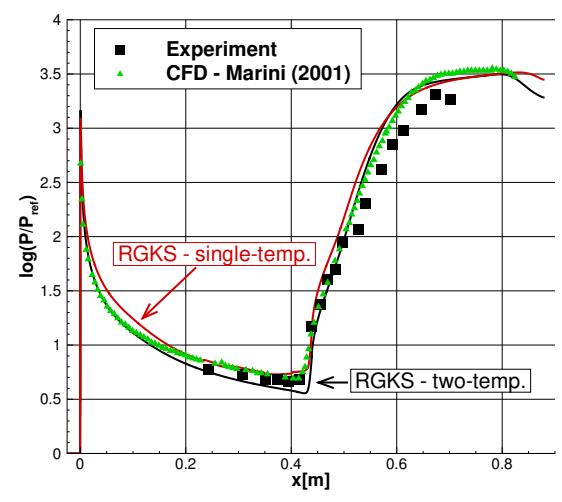

Fig. 2 Compression ramp (CUBRC) - $M_{\infty}=14.1, R e_{\infty} / m=236200$ and $T_{w} / T_{\infty}=3.344$. Comparison of wall pressure (scaled with free-stream pressure) for RGKS solver with experimental data as well as CFD results from Marini (2001).

For the test conditions, the RGKS method with rotational relaxation as well as the version with a single-temperature assumption were used to simulate the flow over the compression ramp. Figure 2 shows the wall pressure (scaled with the free-stream pressure) for these two simulations, compared with the experimental data as well as CFD results from [1]. It can be seen that the proposed RGKS model (with rotational relaxation) generally produces results in better agreement with the experiments than the single-temperature version of the solver. Also, the agreement with the CFD results is generally better. From this comparison, it appears that despite the relatively 

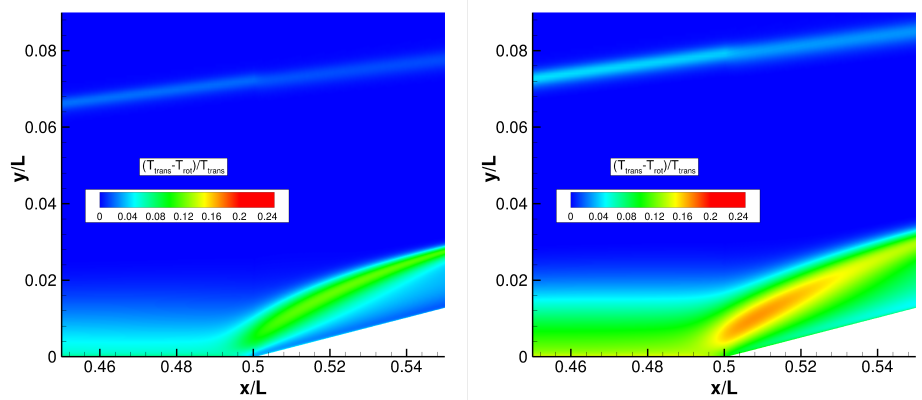

Fig. 3 Compression ramp (CUBRC) - $M_{\infty}=14.1$ and $T_{w} / T_{\infty}=3.344$. Difference in translational and rotational temperature for RGKS simulations at two Knudsen numbers. Flow at experimental test condition, $K n=8.85 \cdot 10^{-5} / \mathrm{m}$ (left) and flow at double Knudsen number (right).

small value of the Knudsen number involved in this test, the inclusion of rotational relaxation as well as a variable rotational collision number in the simulation leads to a more accurate representation of the flow for the gas-kinetic schemes.

The significant contribution from the rotational relaxation can also be seen in the top plot of Figure 3, where the difference between the translational and rotational temperatures in the simulation at the Knudsen of the experiment are shown in the vicinity of the plate/ramp junction. The strongest deviation from thermal equilibrium can be observed in the shock wave/boundary layer interaction region on the ramp. However, a smaller but still significant relaxation takes places in the boundary layer leading up to the shock interaction. This may largely explain the difference in surface pressure between the full RGKS model and the single-temperature version in Figure 2.
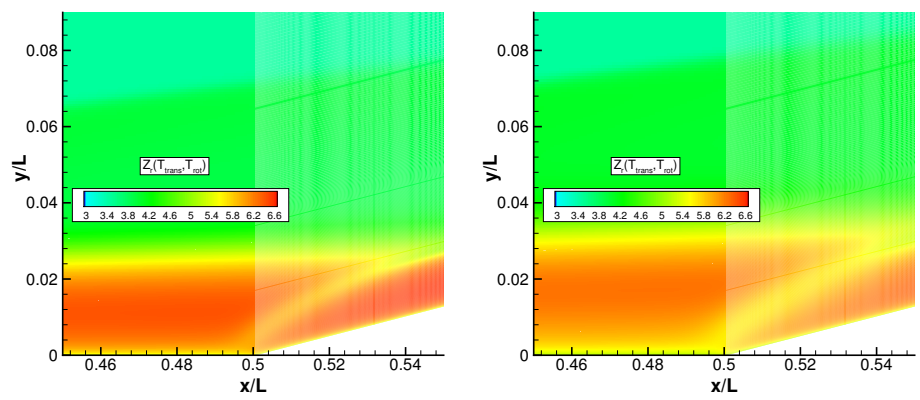

Fig. 4 Compression ramp (CUBRC) - $M_{\infty}=14.1$ and $T_{w} / T_{\infty}=3.344$. Rotational collision number for RGKS simulations. Flow at experimental test condition (left) and flow at double Knudsen number (right). 

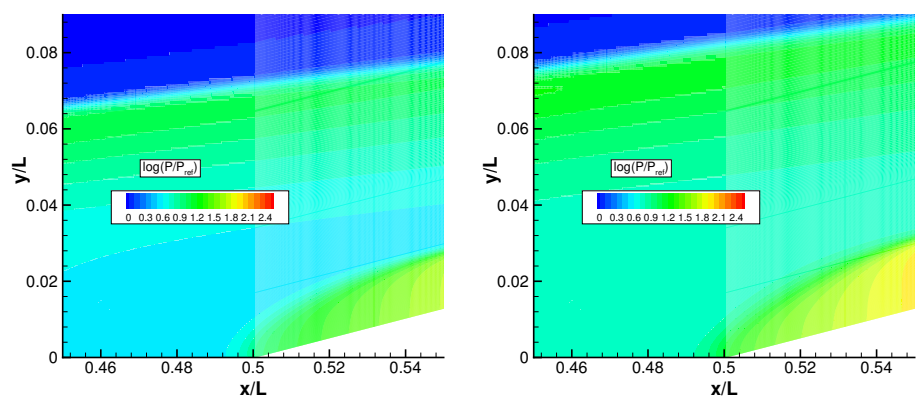

Fig. 5 Compression ramp (CUBRC) - $M_{\infty}=14.1$ and $T_{w} / T_{\infty}=3.344$. Logarithm of scaled pressure (free-stream pressure used as reference) in shock interaction region. RGKS results with rotational relaxation (top) and single-temperature RGKS result (bottom).

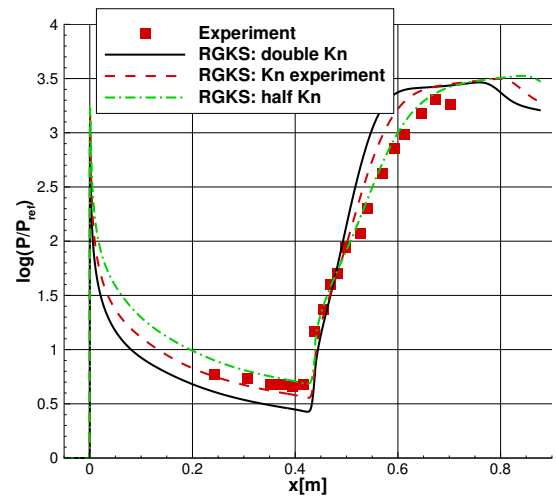

Fig. 6 Compression ramp (CUBRC) - $M_{\infty}=14.1$ and $T_{w} / T_{\infty}=3.344$. Comparison of wall pressure (scaled with free-stream pressure) for RGKS solver for different Knudsen numbers in simulation. For reference, experimental data (at $R e_{\infty} / m=236200$ ) also shown.

In the single-temperature version, a collision number $Z_{r}=5$ was imposed to fix the Prandtl number to 0.711 . For the RGKS results, the $Z_{r}$ from the Valentini model as used in the simulations are shown in Figure 4. In the boundary layers, the collision number is markedly different from 5 , which would further explain differences in the boundary layer development and wall pressures between the RGKS model and its single-temperature version.

Figure 5 shows a comparison of the scaled pressure in the flow field at the conditions of the experiment for the RGKS with rotational relaxation and results from the single-temperature variant of the RGKS. The shock wave generated by the flat plate and its position can be seen to be different in both model due to the different boundary layer development along the flat plate. The pressure in the interaction region is also significantly different with a stronger pressure rise along the ramp for the single-temperature model. 
To assess the sensitivity of the wall pressure to the Knudsen number in this flow, Figure 6 presents a comparison of three sets of results obtained using the RGKS model (with thermal relaxation). The different results are for different Knudsen numbers: the first results is at half the Knudsen number of the experiment, the second result was obtained for the Knudsen number of the experiment and the third result for a Knudsen number increased by two relative to the experiment. For reference, the experimental data is also shown in the figure. Since the Knudsen number changes were created by scaling the length scale of the flow, the Reynolds numbers will also be different for the simulation results. The increased Knudsen number simulation corresponds to the lowest Reynolds number. As could be expected, the wall pressure is already significantly different at the flat plate as a result of the different rate of growth of the boundary layer thickness, leading to a different in the shock wave/boundary layer interaction and the subsequent re-attachment.

From the results presented here, it follows that the strongest thermal relaxation effects results from the difference boundary layer development along the flat plate, which then creates an effect on the shock interaction. The boundary layer on the ramp is less affected by thermal relaxation effects, as a result on the increased density of the flow downstream of the shock and therefore locally small Knudsen number.
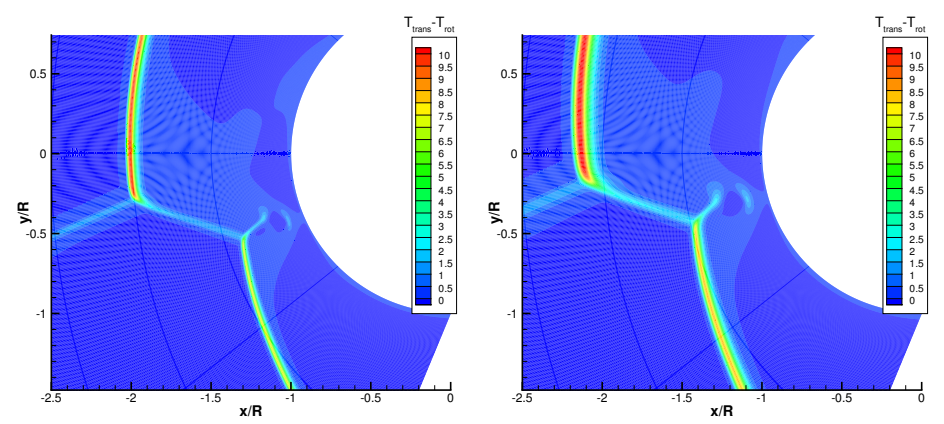

Fig. 7 Edney type IV interaction $-M_{\infty}=9.95$ and $T_{w} / T_{\infty}=5.71$. Difference in translational and rotational temperature for RGKS simulations at two Knudsen numbers. Flow at experimental test condition (left) and flow at double Knudsen number (right).

\section{Edney type IV shock interaction}

The second test case considered is the Edney Type IV interaction investigated experimentally at ONERA Chalais-Meudon as described by [14]. The tests were conducted in the R5Ch blowdown tunnel resulting in a free-stream Mach number of 9.95, free-stream temperature of $52.5 \mathrm{~K}$ and free-stream pressure of $5.9 \mathrm{~Pa}$. The Reynolds number is $166000 / \mathrm{m}$ and the circular cylinder in the test had a radius 

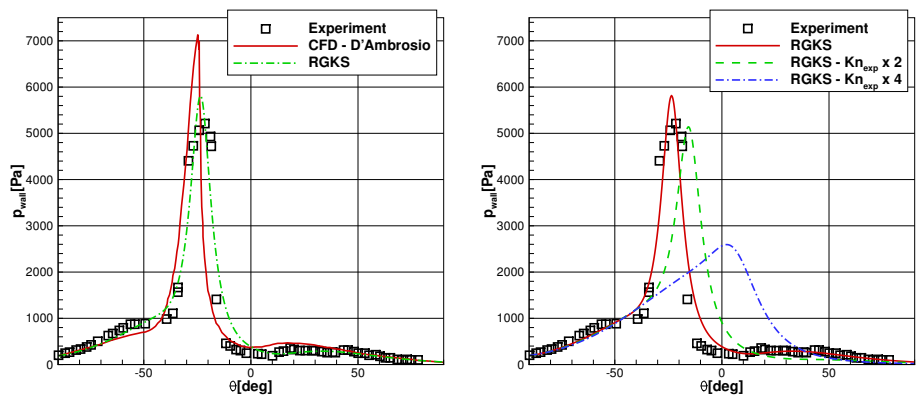

Fig. 8 Edney type IV interaction (experiment at ONERA) - $M_{\infty}=9.95$ and $T_{w} / T_{\infty}=5.71$. Comparison of wall pressure on cylinder (scaled with free-stream pressure) computed with RGKS solver compared with experiments and CFD result from D'Ambrosio (top). Comparison of Knudsen number effect on RGKS solution (bottom).
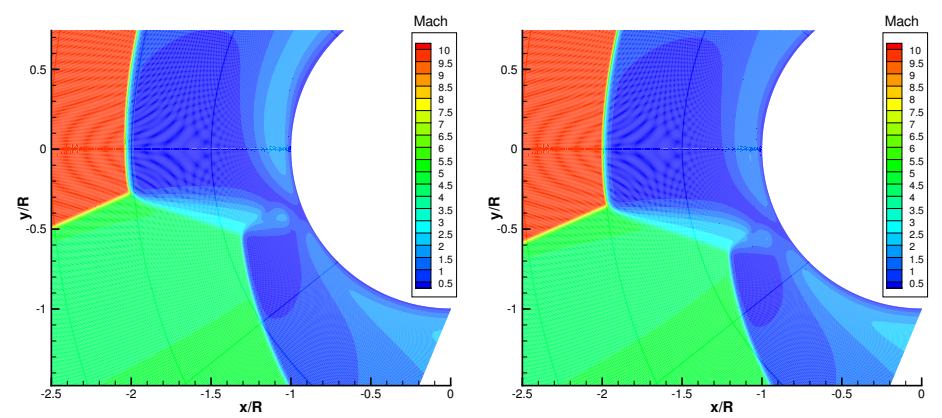

Fig. 9 Edney type IV interaction $-M_{\infty}=9.95$ and model at experimental scale. Mach number contours from RGKS simulations. Different wall temperatures on wedge and cylinder. Flow at experimental test condition, i.e. $T_{w} / T_{\infty}=5.71$ (left) and flow field for $T_{w} / T_{\infty}=3.5$ (right).

of $0.008 \mathrm{~m}$. A ramp at $20^{\circ}$ angle was placed upstream of the cylinder to create an Edney type IV shock interaction. A complicating factor was the ramp extending for $0.050 \mathrm{~m}$ only followed by a flat plate giving rise to a Prandtl-Meyer expansion which in turn curves the oblique shock generated by the ramp before interacting with the bow shock of the cylinder. For this test case, a block-structured mesh with 76 blocks and a total of $10^{6}$ cells in $2 \mathrm{D}$ was employed for the initial results obtained with the RGKS method. Three different Knudsen numbers were considered. The first simulation was conducted at the conditions in the ONERA experiment, while two further simulations involved a scaling down of the flow length scale by a factor of 2 and 4 while maintaining the free-stream conditions. For Knudsen number at experimental conditions and two increased Knudsen numbers, Figure 7 shows the difference in translational and rotational temperatures in the interaction region ahead of the cylinder. As can be expected, the rotational energy relaxation effect is more pronounced for the increased Knudsen number conditions. The plots show the extent of thermal non-equilibrium in the main features of the flow field, i.e. the strong bow shocks as 


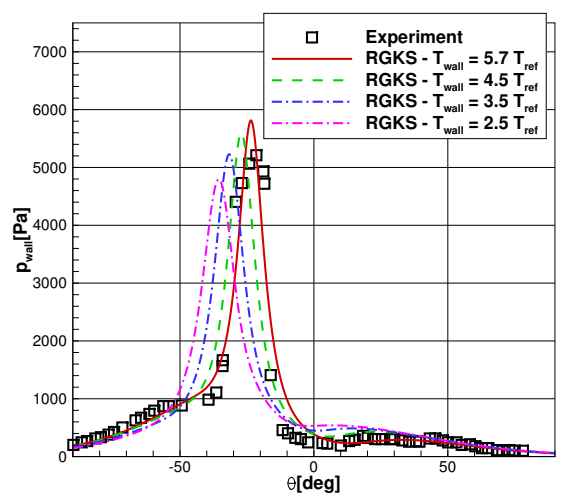

Fig. 10 Edney type IV interaction (experiment at ONERA) - $M_{\infty}=9.95$. Comparison of wall pressure on cylinder (scaled with free-stream pressure) computed with RGKS solver for different wall temperatures imposed on wedge and cylinder compared with experiments (at $T_{w} / T_{\infty}=5.71$ ). Knudsen number in RGKS solution matches that in experiment.

well as the lambda-shock, jet expansion and normal shock just before the jet impinges on the surface. Figure 8 compares the scaled wall pressure on the cylinder as computed with the RGKS method with the experiments from ONERA as well as the previous CFD result from [15]. It can be seen that the agreement of the present results with the experiment is satisfactory in terms of pressure peak and location, with a better agreement than the results from [15]. The convergence behaviour of the current steady-state simulations suggest some flow unsteadiness. This unsteadiness as well as the relatively large pressure tap used in the experiment could partially explain the discrepancy in pressure peak. The figure also shows the effect of increasing the Knudsen number, with an increase (and the associated decrease in Reynolds number) lowering the peak pressure value on the wall as well as moving its location. In addition to the dependency of the shock-interaction on the Knudsen number, the sensitivity of the flow field with respect the imposed wall temperatures was investigated. At the experimental conditions, the wall temperature on the shock-generating wedge as well as the cylinder surface was assumed to the 5.71 times the free-stream temperature. For the selected experimental set-up, a strong type IV interaction was achieved. Here, the experimental set-up in terms of geometry was maintained in the simulations. However, the imposed wall temperature was reduced in steps to 4.5, 3.5 and finally 2.5 times the free-stream temperature. For two different wall temperatures in the simulations, Figure 9 shows Mach number contours in the vicinity of the shock-interaction ahead on the cylinder. It can be see that an important effect is the slightly decreased oblique shock angle for reduced wall temperatures, which can be directly attributed to the reduced growth rate of the boundary layer on the shock-generating wedge, i.e. a reduced boundary-layer displacement effect is present for lower wall temperatures. A second effect of the lower wall temperature is the reduced shock stand-off distance from the cylinder, although this effect 
is relatively small. Clearly, both effects combined lead to a significantly changed shock-interaction with the type IV interaction pattern gradually disappearing for reduced wall temperatures. This can clearly be observed in the wall pressures on the cylinder surface shown in Figure 10 for the different wall temperatures. Clearly, the reduced oblique shock angle moves the interaction region downwards, i.e. towards the negative angles, while the interaction loses strength with decreasing wall temperature. From these results it would follow that for the reduced wall temperature conditions, the shock-interaction intensity could be increased again by moving the shock-generating wedge slightly forward. The presented results clearly demonstrate to the strong dependency of the shock-interaction on both the level of flow rarefaction a fixed Mach (and therefore on Reynolds number) and imposed wall temperatures. In both cases, a significant part of the sensitivity is the result of the changes in oblique-shock location when the wedge-cylinder geometry is left unchanged.

The presented simulation results for the Edney Type IV interaction case were obtained using the Rykov model for the rotational collisional model, as defined in Equation (3). The local value of the rotational collision number has a direct effect on the effective Prandtl number in the continuum limit, as can be seen from Equation (9), as it is a function of translational and rotational temperatures. As discussed previously, a value of 5.0 would give a Prandtl number typically used in a CFD simulation (0.71). One important aspect of the RGKS solver is therefore its ability to model the gas dynamics at a range of conditions for which the constant Prandtl-number assumption is no longer valid, using empirical models or molecular dynamics derived model for the rotational collision number.

\section{Conclusions}

The shock wave/boundary layer interaction on a compression ramp in laminar hypersonic flow was investigated using a gas kinetic scheme derived from the Rykov kinetic model. The effect of flow rarefaction on the shock interaction region and the wall pressure distribution was analysed showing marked differences between the computational model results including rotational relaxation and a simplified approach assuming a single temperature. For the considered conditions, the Knudsen numbers were relatively low. However, in the boundary layer along the flat plate the effect of thermal non-equilibrium was still significant. On the ramp, the boundary layer was less affected by thermal equilibrium effects. The thermal relaxation effect on the wall pressure on the ramp was mainly due to the altered interaction pattern resulting from the different boundary layer development along the flat plate when considering rotational relaxation. Furthermore, the Edney type IV interaction in a hypersonic flow was studied using the RGKS method. The sensitivity of the wall pressure peak and location with increasing Knudsen number was investigated as well as the extent of rotational relaxation in the interaction region. In future works, more detailed investigation of the hypersonic flows with Edney type IV interactions will be conducted. Furthermore, work on hybrid simulations using the gas kinetic 
schemes presented here coupled with the DVM method for the Rykov kinetic model is also ongoing.

Acknowledgements The majority of the results presented were obtained using the EPSRC funded ARCHIE-WeSt High Performance Computer (www.archie-west.ac.uk). EPSRC grant no. $\mathrm{EP} / \mathrm{K} 000586 / 1$.

\section{References}

1. Marini M. (2001) Analysis of Hypersonic Compression Ramp Laminar Flows Under Sharp Leading Edge Conditions. Aerospace Science and Technology 5:257271

2. Deschenes T. and Boyd I. (2011) Extension of a Modular Particle-Continuum Method to Vibrationally Excited, Hypersonic Flows. AIAA Journal 49(9):1951-1959

3. Degond P., Dimarco G. and Mieussens L. (2010) A Multiscale Kinetic-Fluid Solver with Dynamic Localization of Kinetic Effects. Journal of Computational Physics 229:4907-4933

4. Steijl R. and Barakos G. (2012) Computational Fluid dynamics of Partially Rarefied Flows with Coupled Kinetic Boltzmann/Navier-Stokes Methods. ECCOMAS 2012, 10-14 September, Vienna, Austria

5. Xu K. (2001) A Gas-Kinetic BGK Scheme for the Navier-Stokes Equations and its Connection with Artificial Dissipation and Godunov Method. Journal of Computational Physics 171:289-335

6. Xu K. and Huang J.-C. (2010) A Unified Gas-Kinetic Scheme for Continuum and Rarefied Flows. Journal of Computational Physics 229:7747-7764

7. Liu S., Pubing Y., Xu K., and Zhong C. (2014) Unified Gas-Kinetic Scheme for Diatomic Molecular Simulations in all Flow Regimes. Journal of Computational Physics 259:96-113

8. Shakhov E. (1968) Generalization of the Krook Kinetic Relaxation Equation. Mekhanika Zhidkosti i Gaza 3(5):142-145

9. Rykov V. (1975) A Model Kinetic Equation for a Gas with Rotational Degrees of Freedom. Fluid Dynamics 10(6):959-966

10. Colonia S., Steijl R. and Barakos G. (2016) Kinetic Models and Gas Kinetic Schemes for Hybrid Simulation of Partially Rarefied Flows. AIAA Journal 54:1264-1276

11. Steijl R. and Barakos G. (2010) Coupled Navier-Stokes-Molecular Dynamics simulations Using a Multi-physics Flow Simulation Framework. International Journal for Numerical Methods in Fluids 62:1081-1106

12. Valentini P., Zhang C., and Schwartzentruber T. (2012) Molecular Dynamics Simulation of Rotational Relaxation in Nitrogen: Implications for Rotational Collision Number Models. Physics of Fluids 24:106101:1-23

13. Xu K., He X., and Cai C. (2008) Multiple Temperature Kinetic Model and Gas-Kinetic Method for Hypersonic Non-equilibrium Flow Computations. Journal of Computational Physics 227:6779-6794

14. Pot T., Chanetz B., Lefebvre M. and Bouchardy P. (1998) Fundamental Study of Shock-Shock Interference in Low Density Flow: Flowfield Measurements by DLCARS. ONERA TP 1998-140

15. D'Ambrosio D. (2003) Numerical Prediction of Laminar Shock/Shock Interactions in Hypersonic Flow. Journal of Spacecraft and Rockets 40(2):153-161 